\title{
Der Hamburger Entwurf zur Einführung eines
} ärztlichen Ehrengerichts.

In der Hamburgischen Aerzteschaft finden jetzt lebhafte $\mathrm{Er}$ örterungen statt über die Vorlage des Senats, nach welcher eine Abänderung der Aerzteordnung vom 21. Dezember 1894, verbunden mit der Gründung eines ärztlichen Ehrengerichts stattfinden soll Um die Errichtung eines Ehrengerichts waren die Behörden von der Aerztekammer selbst vor einigen Jahren ersucht worden, da die Strafbefugnisse des Vorstandes, welche nur in Verwarnung, Verweis und Wahlentziehung bestehen, nicht ausreichend erschienen gegen solche A erzte, die, gegen moralische Strafen gleichgiltig, nur durch Geldbußen auf den richtigen Weg geleitet werden könnten. Sowohl Bürgerschaft als Senat gingen auf die vorgetragenen Wünsche ein, das Resultat aber aus den vielfachen Vorberatungen beider Körperschaften ist ein derartiges, daß die Hamburger Aerzte mit vollem Recht sich mit Händen und Füben dagegen wehren, mit diesem Danaergeschenk bedacht zu werden. Die Vorlage ist eine in vieler Beziehung verschlechterte Ausgabe des preußischen Ehrengerichtsgesetzes. Während es hier aber wenigstens auf Grund des Umlagerechts gelungen ist, in allen Provinzen ärztliche Unterstützungskassen ins Leben zu rufen, die zum Teil jetzt schon Erhebliches leisten zur Linderung der Not ärztlicher Invaliden und Hinterbliebener, soll in Hamburg die Steuer nur zur Deckung der Kosten der Kammer dienen. - Mehr Erregung rufen die Bestim. mungen hervor, nach welchen nicht nur die beamteten, sondern auch die halbbeamteten Aerzte, auch in solchen Angelegenheiten, welche mit ihrer amtlichen Tătigkeit nichts zu tun haben, nicht vor das ärztliche Ehrengericht gestellt werden dürfen. Und in der Bürgerschaft ist sogar der den preußischen Bestimmungen nachgebildete Antrag abgelehnt wordes, wonach Verfehlungen beamteter Aerzte, die bei den gewöhnlichen Aerzten ein ehrengerichtliches Verfahren nach sich ziehen würden, den Disziplinarbehörden zur Aburteilung angezeigt werden sollen. Diese Verschlechterung gegen die bisherige Einrichtung, bei welcher der Vorstand der Kammer in gleicher Weise beamtete wie nichtbeamtete Aerzte vor sein Forum ziehen konnte, dürfen sich die Hamburger Kollegen keineswegs gefallen lassen. Sie würde dort ebenso einen dauernden Grund zur Milsstimmung abgehen, wie dies in Preußen der Fall ist. - In gleicher Weise ganz unannehmbar ist der Paragraph, wonach nicht nur politische, wissenschaftliche und religiöse Ansichten oder Handlungen, oder die gemeinnlutzige Tätigkeit eines Arztes, sondern auch Vertragsabschlüsse zwischen Aerzten und den Leitern von Heil- und Pflegeanstalten oder den Organen der durch die soziale Versicherungsgesetzgebung geschaffenen Kassen und Anstalten als" solche nicht den Gegenstand eines ehrengerichtlichen Verfahrens bilden können. Danach wärde es in Hamburg nicht straffallig sein, wenn Aerate durch Unterbietungen anders Kollegen aus ihren Kassenstellungen verdrängen oder ihnen bei Kumpfen mit Kassenverwaltungen in den Rücken fallen oder sich durch $\mathrm{Be}$ stechung, Kriechereien und abnliche angenehme Hantierungen die 
Gunst der Kassenvorstände bei Besetzung von Stellen zll erwerben suchen. - Ein anderer Punkt, der: berechtigten Widerspruch hervorruft, ist die Zusammensetzung des Fhrengerichts und Ehrengerichtshofes. Beide bestehen aus je zwei richterlichen Beamten, zu denen beim Ehrengericht drei, beim Ehrengerichtshof fünf ärztliche Mitglieder treten; eine etwaige Verurteilung erfolgt bei jenem mit vier, bei diesem mit sechs Stimmen. Die auffallende Besetzung mit je zwei Richtern erfährt die sonderbare Begründung, daB sonst der Richter durch die ärztlichen Beisitzer überstimnt werden könnte. Welcher Schaden in einem solchen Falle entstehen wïrde, ist uns unklar, wohl aber halten wir die Meinung fïr berechtigt, daß.Aerzte allein entscheiden können, welche Haudlungen gegen die Standesgepflogenheiten und gegen das Standesgefihl verstolsen und da $\beta$ sie hierzu der allwissenden juristischen Oberaufsicht nicht bedürfen. Daß zur Veröffentlichung einer ehrengerichtlichen Verurteilung Eiustimmigkeit erforderlich ist, wird wohl allgemeine Billigung erfahreu. - Die Bestimmung, wonach nicht der Vorsitzende der Aerz.tekammer auch den Vorsitz im Ehreugericht führt, dieser vielmehr auf ein richterliches Mitglied übertragen wird, hängt damit zusammen, daß ein Teil der Ehrengerichtsbarkeit anßer von dem Ehrengerichte nach wie vor auch von dem Kammervorstande ausgeübt werden soll. Letzterer kann auf Terwarnung und Verweis und kleinere Geldstrafen (bis zu 300 M.). das Ehrengericht auf Verwarnung, Гejweis, Geldstrafen bis zu $3000 \mathrm{M}$. und Entziehung des aktiven und passiven Wahlrechts erkennen. Die Berufung gegen Urteile des Torstandes erfolgt an das Ehrengericht. gegen die Urteile des Ehrengerichts an den Ehrengerichtsrat. Wäre nun der Vorsitzende der Aertekammer auch mit dem Vorsitze im Ehrengericht betraut, so würde er bei der Berufung gegen Erkenntnisse mitwirken. die unter seiner Leituug gefaßt worden sind; und das geht natürlich nicht an. Würde es sich aber nicht empfehlen, das ärztliche Mitglied, welches das älteste ist oder welches von den ärztlichen Beisitzern dazı erwählt wird, mit dem Vorsitze zu betranen?

W ir haben in Kürze die weseutlichsten Bestimmungen des Entwurfs den Lesern vorgeführt. Nach ihrer Kenntnisnahme wird es kaum einen Arzt geben, der den Kollegen in Hamburg nicht den dringenden Rat erteilen wird, alles daran zu setzen, um von Fesseln frei zu bleiben, die sich später als sehr drückend erweisen werden. Den Nutzen, welchen man sich von der Einführung der Geldstrafen versprechen könnte, fallt nicht ins Gewicht gegen die Nachteile der Sonderung der Aerzte in zwei Kategorien, der Anfhebung von rein kollegialer Beurteilung und des Zwanges, gegen unehrenhafte Handlungen von Gesetzes wegen nicht einschreiten zu dürfen.

L. Henius (Berlin). 